\title{
On Multi-Robot Area Coverage
}

\author{
Pooyan Fazli \\ Department of Computer Science \\ University of British Columbia \\ Vancouver, B.C., Canada, V6T 1 Z4 \\ pooyanf@cs.ubc.ca
}

\begin{abstract}
Area coverage is one of the emerging problems in multi-robot coordination. In this task a team of robots is cooperatively trying to observe or sweep an entire area, possibly containing obstacles, with their sensors or actuators. The goal is to build an efficient path for each robot which jointly ensure that every single point in the environment can be seen or swept by at least one of the robots while performing the task.
\end{abstract}

\section{Categories and Subject Descriptors}

I.2.9 [Artificial Intelligence]: Robotics - Commercial robots and application; I.2.11 [Artificial Intelligence]: Distributed Artificial Intelligence - Multiagent systems

\section{General Terms}

Algorithms, Performance, Experimentation

\section{Keywords}

Multi-Robot Systems, Teamwork, Coordination, Area Coverage, Offline Coverage, Heterogeneity, Open Systems, Online Coverage, Communication

\section{INTRODUCTION}

Multi-robot area coverage is receiving considerable attention due to its applicability in different scenarios such as search and rescue operations, planetary exploration, intruder detection, environment monitoring, floor cleaning and the like. Several optimization metrics can be considered in building the coverage paths for the robots including time, length of the longest path, sum of the path lengths, initial location of the robots and so on.

There is confusion in the literature regarding the terms Coverage and Exploration. To clarify the problem definition, we note that in exploration, we have an unknown environment and a team of robots is trying to build a map of the area together [2]. In a coverage problem, the map of the environment may be known or unknown and the goal of the team is to jointly observe/sweep the whole area with their sensors or physical actuators. Building a map of the environment is not the ultimate aim of the coverage mission.

Another similar class of problems is Boundary Coverage in which, the purpose of coverage is slightly different from the

Cite as: On Multi-Robot Area Coverage, Pooyan Fazli, Proc. of 9th Int. Conf. on Autonomous Agents and Multiagent Systems ( $A A M A S$ 2010), van der Hoek, Kaminka, Lespérance, Luck and Sen (eds.), May, 10-14, 2010, Toronto, Canada, pp. 1669-1670

Copyright (c) 2010, International Foundation for Autonomous Agents and Multiagent Systems (www.ifaamas.org). All rights reserved. original coverage problem we have defined in this paper. In boundary coverage the aim is to inspect all of the obstacles' boundaries by a team of robots instead of complete coverage of the area [11].

Several research communities including robotics/agents [4], sensor networks [7] and computational geometry [3] work on this class of area coverage problems. In computational geometry, this problem stems from the Art Gallery problem [8] and its variation for mobile guards called the Watchman Route problem [9]. In the Art Gallery problem, the goal is to find a minimum number of static guards (control points) which jointly can cover a work space under different restrictions. On the other hand, in the Watchman Route problem the objective is to compute routes watchmen should take to guard an entire area given only the map of the environment. Most research done on the above problem definitions deal with simple polygons without obstacles, unconstrained guard visibility, and single watchman scenarios.

From a robotics point of view, in a taxonomy presented by Choset [4], the proposed approaches for area coverage are divided into offline methods, in which the map of the environment is known, and online methods, in which the map of the environment is unknown. Choset [4] further divides the approaches for area coverage based on the methods they employ for decomposing the area: Exact Cellular Decomposition, and Approximate Cellular Decomposition.

Previous research on multi-robot area coverage is mostly focused on approaches using the Approximate Cellular Decomposition (e.g. grid-based methods) [1, 12]. These methods have limitations since they do not consider the structure of the environment and have difficulties handling partially occluded cells or covering areas close to the boundaries in continuous spaces. In contrast, methods based on the Exact Cellular Decomposition (e.g. graph-based methods) which employ structures such as the visibility graph for environment representation do not suffer those restrictions [10]. However, while traversing a visibility graph guarantees covering the whole environment in continuous spaces, it might include many redundant movements.

\section{HEALTH CARE FACILITIES: A SAMPLE TASK DOMAIN}

Hospitals, nursing homes and eldercare facilities are suitable environments where robots can help a lot when human care is limited for various reasons such as demographic issues, emergencies, conflicts, lack of funding and so on.

This thesis is motivated by the situations could occur in future health care facilities. Specifically, we are interested in scenarios in which a robot team together monitors the environment (e.g. for fire detection or emergency handling) and the persons living in it (e.g. for tracking patients), and also provide the functionalities necessary in everyday life within those health care facilities (e.g. food delivery). For these 
purposes, the robots need to repeatedly cover the whole target area over time.

\section{THESIS CONTRIBUTIONS}

The following are the expected contributions of the proposed work.

\subsection{Offline Coverage}

We present a new algorithm for covering a known polygonal environment cluttered with static obstacles by a team of mobile robots $[5,6]$. To this end, two environment representation methods called Reduced-CDT and Reduced-Vis based on the Constrained Delaunay Triangulation and the Visibility Graph are introduced so as to model the structure of the target area more efficiently. Also, due to the distributed characteristic of the coverage problem, another algorithm called Multi-Prim's is applied to decompose the graph representing the environment into a forest of partial spanning trees. Each tree is modified through a mechanism called Constrained Spanning Tour to build a cycle which is then assigned to a covering robot. Subsequently, robots start navigating the cycles and consequently cover the whole area. This method has the benefit that it returns the robots to their initial locations, facilitating tasks like collection and storage.

The proposed approach is complete, meaning that every accessible point in the environment will be visited in a finite time. Furthermore, it supports robustness by handling individual robot failure. Our approach has been designed to overcome the restrictive constraint imposed by the robots' limited visibility range as well.

Experiments demonstrate the ability of the robot team to cover the target area based on metrics such as the number of robots in the team, visibility range of the robots, and frequency of robot failure during the coverage mission. We are also going to compare the performance of the proposed approach based on the two methods being used for area representation, i.e. Visibility Graph and the Constrained Delaunay Triangulation.

\subsection{Heterogeneity}

Dealing with robots' heterogeneity is an interesting challenge in this domain. Heterogeneity can be defined in different aspects and contexts, such as different movement capabilities (movement model or velocity constraints) or different task handling abilities. However, the focus of this thesis is to investigate different sensing capabilities in a team of robots. In this variation, the aim is to cover and monitor an environment by using robots with differing visibility ranges; say, one robot could see the objects located in $r 1$-distance while the other robot could recognize the objects located in $r 2$-distance.

\subsection{Open Systems}

We will investigate the situations in which new robots may join the team in the course of the coverage mission. To this end, we propose an algorithm to handle the situation dynamically by efficient task re-allocation among the robots. The goal is to distribute the workload among the team by taking advantage of the new resources (robots) available in the system.

\subsection{Online Coverage}

We will investigate the area coverage task in partially or completely unknown environments, where just local knowledge of the area is provided to the robots. The goal is to present an online, decentralized algorithm for multi-robot area coverage instead of the offline, centralized method discussed earlier.

\subsection{Communication}

We will explore the development of new algorithms to handle communication failure or message loss during the coverage mission. The proposed approaches should also support the case when the robots have a limited range of communication, meaning that a message sent by a robot is transmitted only to robots within a certain distance from that robot.

\section{ACKNOWLEDGMENT}

This work is being supervised by Prof. Alan K. Mackworth at the Computer Science Department of the University of British Columbia.

\section{REFERENCES}

[1] N. Agmon, N. Hazon, and G. A. Kaminka. The giving tree: constructing trees for efficient offline and online multi-robot coverage. Annals of Mathematics and Artificial Intelligence, 52(2-4):143-168, 2008.

[2] W. Burgard, M. Moors, D. Fox, R. Simmons, and S. Thrun. Collaborative multi-robot exploration. In Proceedings. of the IEEE International Conference on Robotics and Automation, ICRA 2000., volume 1, pages 476-481, 2000.

[3] S. Carlsson, B. J. Nilsson, and S. C. Ntafos. Optimum guard covers and m-watchmen routes for restricted polygons. International Journal of Computational Geometry and Applications, 3(1):85-105, 1993.

[4] H. Choset. Coverage for robotics - a survey of recent results. Annals of Mathematics and Artificial Intelligence, 31(1-4):113-126, 2001.

[5] A. Davoodi, P. Fazli, P. Pasquier, and A. K. Mackworth. On multi-robot area coverage. In Proceedings of the 7th Japan Conference on Computational Geometry and Graphs, JCCGG 2009, pages 75-76, 2009.

[6] P. Fazli, A. Davoodi, P. Pasquier, and A. K. Mackworth. Multi-robot area coverage with limited visibility. In Proceedings of The 9th International Conference on Autonomous Agents and Multiagent Systems, AAMAS 2010, 2010.

[7] A. Krause, C. Guestrin, A. Gupta, and J. Kleinberg. Near-optimal sensor placements: maximizing information while minimizing communication cost. In Proceedings of the 5th international conference on Information processing in sensor networks, IPSN 2006, pages 2-10, 2006.

[8] J. O'Rourke. Art gallery theorems and algorithms. Oxford University Press, New York, NY, USA, 1987.

[9] E. Packer. Computing multiple watchman routes. In C. C. McGeoch, editor, 7th International Workshop on Experimental Algorithms, WEA, volume 5038 of Lecture Notes in Computer Science, pages 114-128. Springer, 2008.

[10] I. M. Rekleitis, V. Lee-Shue, A. Peng New, and H. Choset. Limited communication, multi-robot team based coverage. In Proceedings of the IEEE International Conference on Robotics and Automation, ICRA 2004, volume 4, pages 3462-3468, 2004.

[11] K. Williams and J. Burdick. Multi-robot boundary coverage with plan revision. In Proceedings of the IEEE International Conference on Robotics and Automation, ICRA 2006., pages 1716-1723, 2006.

[12] X. Zheng, S. Jain, S. Koenig, and D. Kempe. Multi-robot forest coverage. In Proceedings of the IEEE/RSJ International Conference on Intelligent Robots and Systems, IROS 2005, pages 3852-3857, 2005. 